\title{
Short form 1b human prolactin receptor down-regulates expression of the long form
}

\author{
Dunyong Tan and Ameae M Walker \\ Division of Biomedical Sciences, University of California, Riverside, California 92521, USA \\ (Correspondence should be addressed to A M Walker; Email: ameae.walker@ucr.edu)
}

\begin{abstract}
Alternative splicing produces different human prolactin (PRL) receptors. These include a long form (LF) and two short forms (SF1a and SF1b). The SFs of the receptor can act as dominant negatives for PRL effector function through the LF. This is proposed to be due to LF-SF heterodimerization and resultant interference with LF-LF dimer signaling. We, along with others, have provided evidence for LF-SF heterodimerization of the human receptors in support of this mechanism, along with others. However, to further investigate the ways SF may influence LF function, we co-transfected human embryonic kidney 293 cells with vectors coding for tagged (green fluorescent protein (GFP) or luciferase) LF alone or plus untagged SF1b and measured LF-GFP intensity, LF-luciferase activity, and LF mRNA $48 \mathrm{~h}$ later. Equal amounts of SF1b cDNA decreased LF-GFP fluorescence intensity, LF-luciferase activity, and LF mRNA by $80-100 \%$. Similar co-transfections with untagged LF had no significant effect on tagged LF expression. Use of hygromycin showed degradation of already formed protein was the same for LF-luciferase alone and LF-luciferase with SF1b. Inhibition of mRNA synthesis, on the other hand, showed that SF1b expression accelerated LF mRNA degradation two- to three-fold. SF1b also down-regulated expression of endogenous LF mRNA in T47D breast cancer cells and opposed an increase in cell number resulting from transfection with extra LF alone. These results demonstrate a previously unrecognized mechanism whereby SF1b affects the end result of signaling through the LF receptor. The effects on cell number also support the concept that the LF:SF1b ratio may be relevant to tumor growth.
\end{abstract}

Journal of Molecular Endocrinology (2010) 44, 187-194

\section{Introduction}

Multiple different isoforms of the human prolactin (PRL) receptor have been described (Kline et al. 1999, Hu et al. 2001, Trott et al. 2003, Tan et al. 2008), most of which are produced by alternate splicing of a single gene transcript. The most abundant varieties include a long form (LF), which is 598 amino acids in length, an 'intermediate' form of 325 amino acids and two short forms of 352 (SFla) and 264 (SF1b) amino acids. Different abundances and ratios of these forms have been associated with disease. For example, increased LF PRL receptor expression is associated with early prostate disease (Leav et al. 1999), and activation of signaling molecules downstream of the LF PRL receptor is associated with higher prostate tumor grade (Li et al. 2004). In the mammary gland where PRL has also been suggested to play a role in disease, breast cancer progression is associated with a decreased ratio of short to long PRL receptors (Meng et al. 2004). This progression has been attributed to loss of a dominant negative effect of the short receptors on signaling through the LF receptor, a dominant negative effect proposed to be produced by heterodimerization of short and long receptors (Trott et al. 2003,
Qazi et al. 2006). Studies from this lab (Tan et al. 2005) and the Dufau lab (Qazi et al. 2006) have demonstrated that both homo- and hetero-dimers of long and short receptors are formed, and that ligands interact with both (Tan et al. 2005). The only pairing of these receptors that does not seem to occur is between the two different SFs (Tan et al. 2005).

PRL belongs to a large cytokine family whose members utilize single transmembrane domain receptors with associated, rather than intrinsic, tyrosine kinases (Cosman 1993). The traditional view has been that members of this cytokine family bring two (or more) receptors together, allowing transphosphorylation of the associated tyrosine kinases. It now seems that many (if not all) receptors are pre-dimerized and that ligands induce a change in conformation of the receptors which brings the tyrosine kinases together (Grotzinger 2002, Tan et al. 2005, Walker 2005, Qazi et al. 2006). Most of what we know about PRL receptor signaling comes from analysis of the LF, where the best studied pathway is activation of Jak2 and Stat 5 . After transphosphorylation of two LF-associated Jak2 molecules in response to ligands, Jak2 phosphorylates the receptor, allowing for more efficient association between Stat5 and the receptor (Clevenger et al. 2001).

DOI: 10.1677/JME-09-0101 Online version via http://www.endocrinology-journals.org 
Docked Stat5 is then phosphorylated by Jak2, phosphorylated Stat5 leaves the receptor, dimerizes and enters the nucleus where it acts as a transcription factor.

Association of Jak2 with the receptor is influenced by the presence of two intracellular regions, boxes 1 and 2. Box 1 is absolutely required for Jak2 activation (Clevenger et al. 2001), while the importance of box 2 is debated (Clevenger et al. 2001). Based on the presence of box 1 in both the short receptors, one would predict that both would have associated Jak2. This prediction is in agreement with data in a recent publication from the Dufau lab that describes the degree of activation of Jak2 (fold basal) by PRL as essentially the same for all three forms of the receptor (Qazi et al. 2006). However, based on the fact that the short receptors do not have the specific tyrosine in the LF receptor normally phosphorylated by Jak2 (Clevenger et al. 2001), one would predict that neither SF would be able to efficiently phosphorylate Stat5, although to our knowledge this has not yet been specifically examined. What has been examined is the downstream effect of reduced Stat5 phosphorylation, quantified by activation of a Stat5-responsive promoter 24 to $48 \mathrm{~h}$ after receptor transfection (Qazi et al. 2006). There is a lot of time therefore between signal generation and the downstream effect measured, time during which additional factors could influence reporter activation. One such additional factor is described in the current manuscript, where we show that co-expression of the SF1b receptor decreases the amount of LF mRNA by accelerating LF mRNA degradation.

\section{Materials and methods}

\section{Materials}

The PRLR cDNAs, encoding the LF, SFla and SF1b were originally kindly provided by $\mathrm{Dr} \mathrm{B} \mathrm{K}$ Vonderhaar (NCI, Bethesda, MD, USA) and were cloned into the green fluorescent protein (GFP) and Rluc vectors as described previously (Tan et al. 2005). A $\beta$-casein-Luc plasmid, which contains $2.4 \mathrm{~kb}$ of the $\beta$-casein promoter upstream of a luciferase reporter gene (Lee et al. 1989, Brockman \& Schuler 2005), was a generous gift from Dr Linda Schuler (Madison, WI, USA) and Jeffrey Rosen (Baylor, TX, USA). The cell number assay reagents (3-(4, 5-dimethylthiazol-2-yl)-5-(3-carboxymethoxyphenyl)-2-(4-sulfophenyl)-2H-tetrazolium (MTS), inner salt) and phenazine methosulfate (PMS) were products of Promega. Reverse transcriptase and Pfu polymerase were purchased from Stratagene (La Jolla, CA, USA). The transfection reagents were lipofectamine 2000 (Invitrogen) or FuGENE HD (Roche Applied Sciences). Unmodified PRL was purified from Escherichia coli (BL21) as described previously
(Chen et al. 1998). pRed-tandem, which was used as a non-specific protein-expressing vector to keep amounts of transfected DNA constant, was obtained from Evrogen through Axxora (San Diego, CA, USA).

\section{Cell culture and transfection}

Human embryonic kidney 293 (HEK293) cells were maintained in DMEM (Invitrogen) containing high glucose, $1 \mathrm{mM}$ sodium pyruvate and pyridoxine hydrochloride, $2 \mathrm{mM}$ L-glutamine, supplemented with $10 \%$ (v/v) fetal bovine serum (Invitrogen), 100 units $/ \mathrm{ml}$ penicillin, and $0.7 \mu \mathrm{M}$ streptomycin. Lipofectamine 2000 was used for transfection. The two breast cancer epithelial cell lines, T47D and MCF-7, were cultured in RPMI 1640 medium, supplemented with $10 \%$ (v/v) fetal bovine serum, 100 units $/ \mathrm{ml}$ penicillin, and $0.7 \mu \mathrm{M}$ streptomycin. Transient transfections were performed the day after plating (cells $90-95 \%$ confluent) using FuGENE HD in accordance with the protocol provided by the vendor. Amounts of transfected DNA were held constant by using a non-specific vector, pRed-tandem. This vector expresses a far red fluorescent protein using the cytomegalovirus (CMV) promoter. It therefore also serves to control for artifacts produced by the taxation of the protein synthetic machinery when overexpressing proteins. Further details of specific experiments are given in the figure legends.

\section{RNA isolation, RT-PCR}

Forty-eight hours (or as indicated for the time course experiment) after transfection, the cells were washed three times with Dulbecco's PBS (DPBS), and RNA extractions were performed using TRIzol reagent (Invitrogen). Total RNA was used for first-strand synthesis of cDNA using reverse transcriptase. Briefly, $5 \mu \mathrm{g}$ total RNA and $3 \mu \mathrm{l}$ random primer (Epicentre, Madison, WI, USA) in nuclease-free water were incubated at $65^{\circ} \mathrm{C}$ for $5 \mathrm{~min}$, then slowly cooled to room temperature to allow the primers to anneal to RNA. Then, $5 \mu \mathrm{l}$ of $5 \times$ RT buffer, $1 \mu \mathrm{l}$ of RNase inhibitor, $2 \mu \mathrm{l}$ of $100 \mathrm{mM}$ dNTP mix, $1 \mu \mathrm{l}$ of reverse transcriptase (Stratagene) were added and mixed and incubated at $42{ }^{\circ} \mathrm{C}$ for $1 \mathrm{~h}$. For PCR, $1 \mu \mathrm{l}$ of RT product was added to a $50 \mu \mathrm{l}$ reaction mixture containing $5 \mu \mathrm{l}$ of $10 \times \mathrm{Pfu}$ polymerase buffer, $4 \mu \mathrm{l}$ of dNTP $(4 \mathrm{mM})$, $2.5 \mu \mathrm{l}$ of forward or reverse primer $(10 \mathrm{mM})$ and $1 \mu \mathrm{l}$ of Pfu polymerase. LF mRNA was detected using two different sets of primers: $5^{\prime}$-GAC ACG CGT ACC ATG AAG GAA AAT GTG-3' (forward) and 5'-AAC GGT ACC A GTG AAA GGA GTG TGT-3' (reverse), which yield a $1869 \mathrm{bp}$ fragment of LF open reading frame or $5^{\prime}$-GAT TTG ATG CTC ATC TGT TGGA- ${ }^{\prime}$ (forward) and 5'-TCC AGG TAT GTG GGT TTC AT-3' (reverse), which 
yield a $199 \mathrm{bp}$ fragment of the LF open reading frame. The first set of primers uses one primer that is common to all receptors. To ensure that effects observed on mRNA levels were not produced by competition among primers, experiments were repeated with LF-specific primers (the second set). PCR parameters were as follows: $95{ }^{\circ} \mathrm{C}$ for $1 \mathrm{~min}, 55^{\circ} \mathrm{C}$ for $50 \mathrm{~s}, 72{ }^{\circ} \mathrm{C}$ for $2 \mathrm{~min}$, with a final extension period of $72{ }^{\circ} \mathrm{C}$ for $10 \mathrm{~min}$. Cycle titration ensured a semi-quantitative result. All data were normalized to $\beta$-actin mRNA. Reactions for realtime PCR (conducted with both sets of primers) were performed on an ABI Prism 7700 Sequence Detection System by utilizing $2 \times$ SYBR Green PCR Master Mix (4309155, Applied Biosystems, Foster City, CA, USA) according to the product manual. The data analysis was carried out as described in User Bulletin \#2 of ABI Prism 7700 Sequence Detection System.

\section{Viable cell number assay (MTS assay)}

This assay was performed in the linear range for T47D cells under stringent conditions previously described (Huang et al. 2004), including removal of serum prior to the addition of the MTS reagent. Briefly, $42 \mathrm{mg}$ of MTS reagent powder was dissolved in $21 \mathrm{ml}$ DPBS and filter-sterilized using a $0 \cdot 2 \mu \mathrm{m}$ filter. To this $100 \mu \mathrm{l}$ PMS/ $2 \mathrm{ml}$ MTS solution was added under light-protected conditions. Twenty microlitres of the freshly combined MTS/PMS solution were added to each well of a 96-well assay plate containing $100 \mu \mathrm{l}$ of cells in culture medium without serum. The cells plus reagents were then incubated at $37^{\circ} \mathrm{C}, 5 \% \mathrm{CO}_{2}$ for $3 \mathrm{~h}$, after which time the absorbance at $490 \mathrm{~nm}$ was recorded using a microplate spectrophotometer.

\section{Luciferase assay}

After transfection and expression of a luciferase construct, cells cultured in 96-well plates were washed three times with DPBS, and then reporter lysis buffer (Promega) was added to the plate. To ensure complete lysis, a freeze-thaw was performed followed by 15 -min of shaking at room temperature. Luminescence was measured after the addition of substrate $(50 \mu \mathrm{l} /$ well of luciferin as provided by Promega).

\section{Statistical analysis}

Each experiment was conducted three times. Replicates within an experiment varied from 3 (real-time RT-PCR) to 12 (luciferase analyses). Statistical significance was largely determined by $t$-test, with correction for multiple comparisons by the method of Bonferroni. The adequacy of the straight line fit over time for data was verified using the classic ANOVA lack-of-fit test.
The equality of slopes across the groups was assessed by testing for interaction between the slope and the group effects. All assumptions needed to justify these tests were verified by $Q Q$ plot for normality and Bartlett's test for homogeneity of variance.

\section{Results}

Others have demonstrated that co-expression of human $\mathrm{LF}$ and SF1b receptors reduces $\beta$-casein promoter activation in response to PRL versus cells expressing LF alone (Trott et al. 2003, Qazi et al. 2006). Figure 1 shows the result of a similar experiment using our receptor vectors. It is clear that co-transfection with an equal amount of SF1b cDNA markedly decreased $\beta$-casein-firefly luciferase promoter activity in response to PRL versus LF alone. In this experiment and all the subsequent experiments presented, the amount of transfected DNA was the same for each experimental group, made equal with an irrelevant, but proteinexpressing vector. In addition, the different lengths of the receptor cDNAs within the vector were corrected for when stating the transfection ratios.

To further investigate the potential roles of the SF1b receptor, we initiated a series of experiments, the results of which led to the hypothesis that SF1b decreased the amount of the LF receptor. To test this hypothesis, we first examined the effect of SF1b co-transfection on the expression of GFP-tagged LF. Figure 2A shows the result of one such experiment where an equal amount of transfected SF1b cDNA was or was not co-transfected with LF-GFP ${ }^{2}$ cDNA. The graph illustrates the fluorescence emission for LF-GFP ${ }^{2}$ protein. Three curves for each condition are illustrated. The area under the

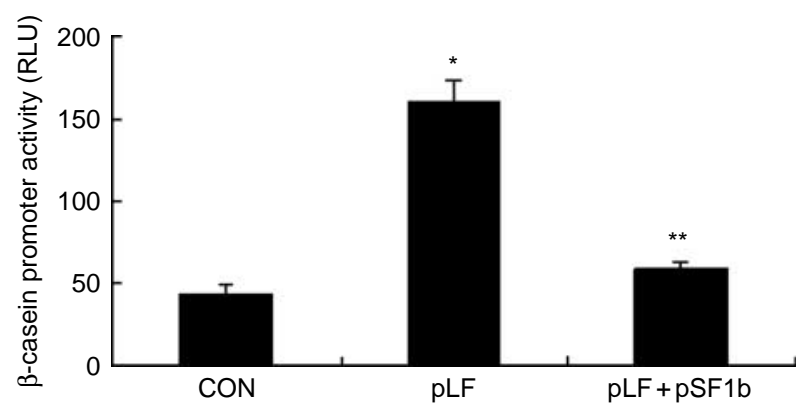

Figure $1 \mathrm{SF} 1 \mathrm{~b}$ inhibits LF-induced activation of the $\beta$-casein promoter. HEK293 cells were transiently transfected with a plasmid containing a $2.4 \mathrm{~kb}$ portion of the $\beta$-casein promoter (tagged with firefly luciferase) alone (CON) or together with LF CDNA or LF cDNA plus SF1b cDNA. Amounts of DNA were made constant with pRed-tandem (Evrogen Axxora, San Diego, CA, USA) that causes the expression of a fluorescent red protein. Cells were incubated for $48 \mathrm{~h}$ in the presence of unmodified PRL $(500 \mathrm{ng} / \mathrm{ml})$, after which the $\beta$-casein luciferase activity was measured. ${ }^{\star} P<0.01$ versus $\mathrm{CON}$; ${ }^{\star \star} P<0.01$ versus pLF. 

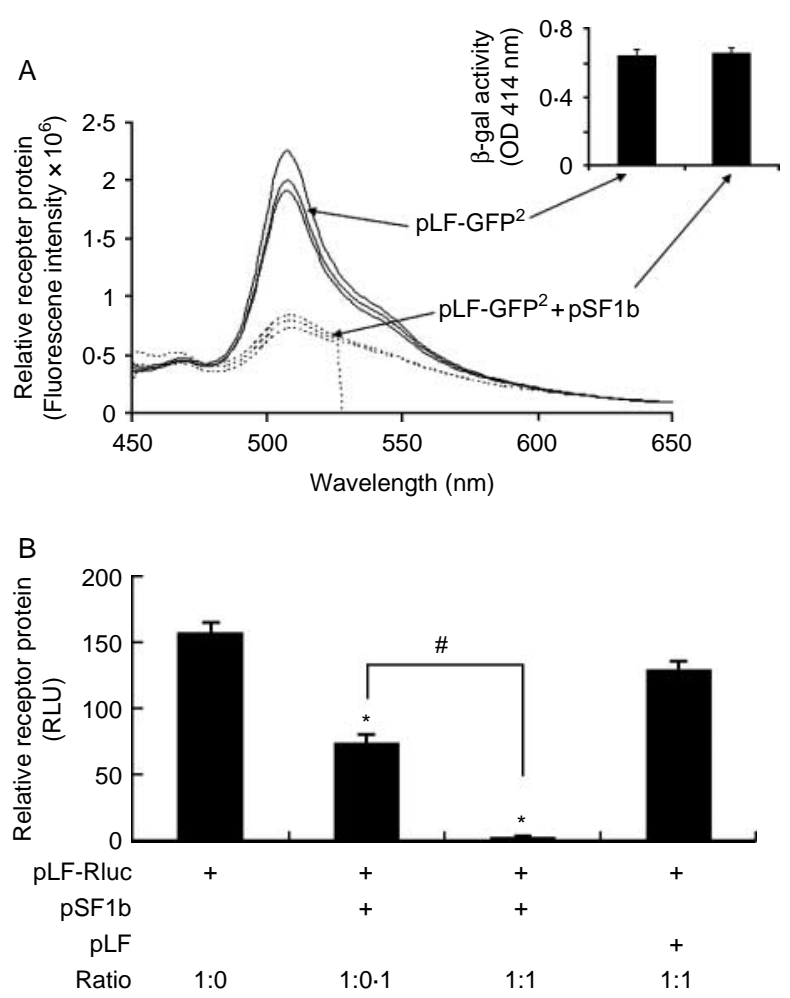

Figure 2 Down-regulation of LF protein by co-expression of SF1b. HEK 293 cells were transiently transfected with a vector coding for the LF receptor with a GFP tag (pLF-GFP ${ }^{2}$ ) alone or with this plus a vector coding for the SF1b receptor ( $\mathrm{pSF} 1 \mathrm{~b}$ ) (panel $A$ ) or with a vector coding for the LF receptor tagged with luciferase (pLF-Rluc) alone or this plus a vector coding for untagged LF (pLF-Rluc + pLF) or SF1b (pLF-Rluc+pSF1b) (panel B). Amounts of DNA were made constant with pRed-tandem. Forty-eight hours post transfection, the green fluorescence was scanned using a fluorospectrometer, or luciferase activity was determined, as appropriate. For GFP ${ }^{2}$, the emission maximum is $510 \mathrm{~nm}$. The inset shows equivalent transfection efficiency, as measured by $\beta$-galactosidase activity, for the two conditions shown. ${ }^{*} P<0.01$ versus cells transfected with pLF-Rluc alone; ${ }^{\#} P<0.01$.

specific part of the spectral curve is reduced by $80 \%$ with co-transfection. Co-transfection did not alter transfection efficiency of the LF-GFP ${ }^{2}$, as illustrated in the inset where co-transfected $\beta$-galactosidase activity is shown. Similar experiments with untagged LF showed no reduction of LF-GFP ${ }^{2}$ fluorescence (data not shown here since similar results are shown in Fig. 2B).

To eliminate the possibility that this might be a phenomenon related to the GFP tag on the LF receptor, we then conducted a similar experiment using Renilla luciferase as the tag for the receptor protein. The added advantage of the luciferase tag is increased sensitivity combined with ease of quantification. Figure 2B shows the effect of one-tenth and equal amounts of SF1b cDNA on the expression (as judged by enzyme activity) of LF-Rluc protein. SF1b, transfected at one-tenth the amount of LF-Rluc, reduced luciferase activity by half, and equal amounts essentially eliminated all activity. Equal amounts of transfected untagged LF on the other hand had little effect on luciferase activity.

To test whether SF1b was affecting degradation of the $\mathrm{LF}$ receptor, we transfected the cells, allowed them to express protein (LF-Rluc) and then examined the amount of luciferase activity as a function of time after the addition of the protein synthesis inhibitor, hygromycin B (Fig. 3). To be able to combine experiments, the results are presented as percent of the value at the time of hygromycin administration. Interestingly, the luciferase activity, and by inference therefore the amount of LF receptor protein, was very stable in the control cells without hygromycin B during the 24-h observation period. This suggests that under normal circumstances, synthesis is carefully balanced by degradation despite the fact that transcription was under the influence of a strong CMV promoter. The amount of LF-Rluc activity decreased at a constant rate in the hygromycin-treated samples such that $80 \%$ was gone by $24 \mathrm{~h}$, indicative of a significant degradation rate. However, the rate of degradation of already formed protein was unaltered in the presence of SF1b.

The most likely explanation for the effect of co-expression with SF1b was therefore on levels of mRNA for the LF. Figure 4A shows the result of semiquantitative RT-PCR examining the relative steady state level of mRNA for the LF of the receptor with and without co-transfection with equal amounts of SF1b. SF1b was effective at decreasing the amount of LF mRNA by about $90 \%$. To add an additional control, the effect of co-transfection with SFla was also analyzed. Unlike SF1b, this had no effect on LF mRNA levels.

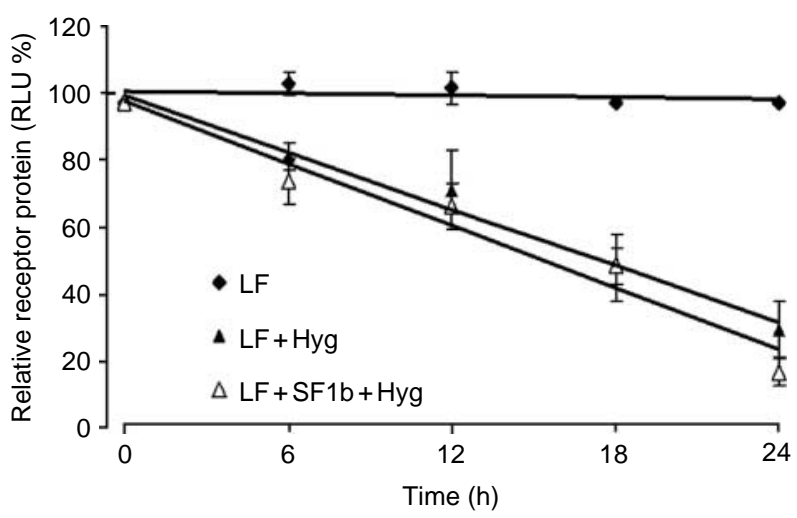

Figure $3 \mathrm{SF} 1 \mathrm{~b}$ has no significant effect on the rate of already formed LF-Rluc protein degradation. HEK293 cells were transiently transfected with pLF-Rluc alone or pLF-Rluc plus pSF1b. Amounts of DNA were made constant with $\mathrm{pRed}$-tandem. Twelve hours post transfection, hygromycin $B(50 \mu \mathrm{g} / \mathrm{ml})$ was added into some wells (as indicated in the figure). Luminescence was measured at $0,6,12,18$ and $24 \mathrm{~h}$ post administration of hygromycin and expressed as a percentage of the value at $0 \mathrm{~h}$ (when hygromycin B was added). 


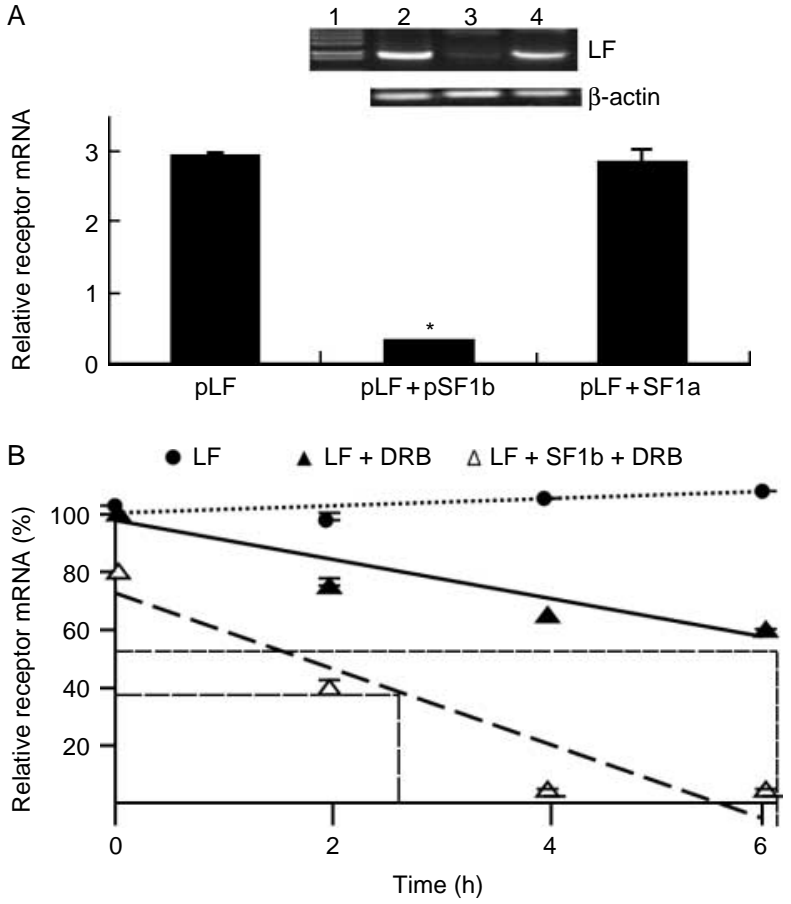

Figure 4 Down-regulation of LF mRNA by co-expression of SF1b. For panel A, HEK293 cells were transiently transfected with pLF or this plus $\mathrm{pSF} 1 \mathrm{~b}$ or $\mathrm{pSF} 1 \mathrm{a}$ (LF to SF1b cDNA ratios of $1: 1$ ). Amounts of DNA were made constant with pRed-tandem. Fortyeight hours post transfection, total RNA was extracted for semiquantitative RT-PCR. The upper panel in 'A' shows an example of an ethidium bromide-stained gel showing the LF and $\beta$-actin amplicons: 1, bp ladder; 2, LF alone; 3, LF+SF1b; 4, LF+SF1a. Data in the lower panel of ' $A$ ' are normalized to $\beta$-actin mRNA. ${ }^{\star} P<0.01$ versus $p L F$. For panel $B$, HEK 293 cells were transiently transfected with pLF or pLF + SF1b. Amounts of DNA were made constant with pRed-tandem. Twelve hours post transfection, the medium was changed to DMEM containing $10 \%$ FBS with or without $40 \mu \mathrm{M}$ DRB, as indicated in the figure. Total RNA was prepared at 0, 2, 4 and $6 \mathrm{~h}$ after a wash in serum-free medium and real-time qPCR was performed. Data were normalized by $\beta$-actin mRNA. The time taken for degradation of $50 \%$ of the mRNA value at $\mathrm{O} h$ is indicated by dashed lines for the samples with DRB, with and without SF1b.

To determine whether this was an effect on mRNA stability, we transfected the cells, allowed them to express mRNA and then examined the amount of LF receptor mRNA by real-time RT-PCR as a function of time. Figure $4 \mathrm{~B}$ shows that when LF alone is transfected, there is a very consistent level of LF mRNA expressed over the time-period examined, again illustrating tight control overexpression of the LF receptor. After addition of the transcriptional inhibitor, 5,6-dichlorobenzimidazole riboside (DRB), there was a steady rate of decline in mRNA levels such that about half was gone in a $\sim 6$-h period. Co-transfection with SF1b decreased expression of the LF in the period prior to the addition of DRB and hence the 0-h value is below the other two on the graph. In the presence of DRB, SF1b then accelerated the decrease in mRNA levels such that half the 0 -h value was gone in $2.5 \mathrm{~h}$. Since, a) one needs to allow for transcription prior to the administration of DRB in order that there is LF mRNA to be followed and b) without DRB one doesn't know whether the effect is on transcription or degradation, it is not possible to look at earlier time points where one would expect the "plus SF1b and DRB' line to intersect the 'LF alone' line.

The preceding results were the outcome of co-transfection of two vectors into a cell line that does not normally express PRL receptors. We therefore examined whether transfection with SF1b would have the same effect on two cell lines that normally express high levels of LF receptor, the human breast cancer cell lines T47D and MCF7. In this case, cells were transfected with increasing amounts of SF1b cDNA and endogenous LF mRNA was examined after $48 \mathrm{~h}$. Figure 5 shows a decrease in the expression of LF mRNA in T47D cells. A similar result was obtained in MCF7 cells (data not shown).
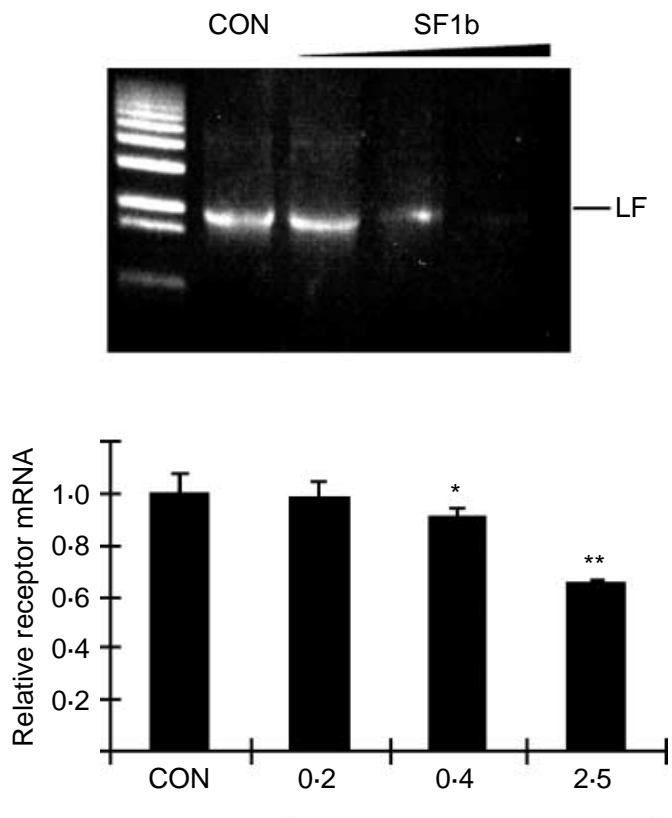

Amount of SF1b ( $\mu$ g vector DNA)

Figure 5 Effect of increased expression of SF1b on endogenous LF mRNA in T47D cells. Increasing amounts of SF1b cDNA were transiently transfected into T47D cells $(0.2-2.5 \mu \mathrm{g})$, and the effect on LF mRNA was measured $48 \mathrm{~h}$ later. Amounts of transfected DNA were made constant with pRed-tandem. The top panel shows an example of the ethidium bromide-stained gel showing the LF amplicon, and the bottom panel shows real-time RT-PCR data normalized to $\beta$-actin mRNA. Control (CON) cells were those transfected with pRed-tandem only. ${ }^{\star} P<0.05$ and ${ }^{* \star} P<0.01$ versus CON. Transfection efficiency was the same for each group and resulted in the transfection of $27 \pm 3.1 \%$ of cells. The data presented are not corrected for the percentage of cells transfected. 


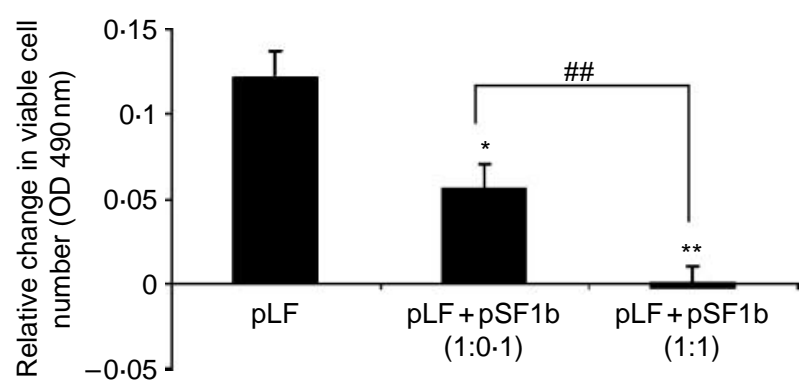

Figure 6 Effect of increased expression of LF receptor or this plus SF1b on T47D cell number. Twelve hours post transfection with $\mathrm{pLF}$, or this plus one-tenth or equal amounts of pSF1b, the medium was changed to RPMI 1640 without FBS. An MTS assay, which reflects viable cell number as OD $490 \mathrm{~nm}$, was performed after a further 5-day incubation in the presence of unmodified PRL $(500 \mathrm{ng} / \mathrm{ml})$. FBS is removed and PRL is provided to make cell survival/proliferation dependent on PRL-PRLR interactions. Amounts of DNA were made constant with pRed-tandem. The control value (OD $490 \mathrm{~nm}$ in cells transfected with pRed-tandem and treated with $P R L$ ) was subtracted to illustrate the change in response to transfection with the extra receptors and to normalize for transfection efficiency. ${ }^{\star} P<0.05,{ }^{\star \star} P<0.01$ versus $\mathrm{CON}$; ${ }^{\# \#} P<0.01$ versus LF.

To determine whether this effect on LF mRNA had any functional consequences and to do so in such a way that we could examine the amount of SF1b versus LF cDNA required and correct for transfection efficiency, we conducted the experiment shown in Fig. 6. For this experiment, T47D cells were transfected with cDNA coding for the LF, or with this plus one-tenth or equal quantities of cDNA coding for SF1b. After a recovery period of $12 \mathrm{~h}$, cells were placed in serum-free medium and incubated for 5 days in the presence of PRL. To illustrate the changes wrought by transfection with the receptor-expressing cDNAs, the value generated by the control cells was subtracted. Results are expressed as OD $490 \mathrm{~nm}$ (relative viable cell number) at the end of the 5-day incubation. Transfection with cDNA coding for the LF increased cell number over the control. Cotransfection with one-tenth or equal amounts of SF1b offset this increase by $\sim 50$ and $\sim 100 \%$ respectively.

\section{Discussion}

Others have demonstrated a dominant negative effect of expression of the short human PRL receptors on LF receptor function using $\beta$-casein promoter-luciferase constructs (Trott et al. 2003, Qazi et al. 2006), a dominant negative effect that has so far been attributed to heterodimerization of SF and LF receptors. We, along with others, have contributed to the evidence in favor of such heterodimerization (Tan et al. 2005, Qazi et al. 2006). SF and LF receptors have been shown to heterodimerize in overexpression systems, where their interaction can be followed in live cells (Tan et al. 2005, Qazi et al. 2006), and other homo- and hetero-pairs of receptors have been shown in normal cells where their association at physiological levels of expression can be demonstrated by co-immunoprecipitation (Gadd \& Clevenger 2006). There is no doubt therefore that heterodimerization occurs. Such heterodimerization is predicted to reduce Stat 5 activation in response to PRL, and it is this reduction in Stat5 activation that is presumed to reduce activation of the Stat 5 responsive promoter of $\beta$-casein. However, at issue is whether or not this explains all of the dominant negative effect observed, especially since measurement of the effect occurs some $48 \mathrm{~h}$ after transfection and after $24 \mathrm{~h}$ of stimulation with PRL.

Using a $2 \cdot 4 \mathrm{~kb}$ portion of the $\beta$-casein promoter for the promoter activity read-out, we have previously shown that co-transfection with SFla can reduce the degree to which PRL is able to activate the promoter through the LF, but also that when used alone, SF1a is a less efficient, but effective activator (Tan et al. 2005, 2008). This is not true of SF1b, which in terms of $\beta$-casein promoter activity is only a dominant negative to the LF (Tan et al. 2005). For the current study, we have therefore focused on SF1b.

Using two different tags, added to the receptor during translation to follow LF receptor protein, both of which have previously been shown to have no adverse effect on the ability of the LF receptor to activate a $\beta$-casein promoter (Tan et al. 2005), we have demonstrated that co-transfection with equal amounts of SF1b reduced LF protein expression by $80-100 \%$ in a 48 -h period. Because we obtained the same result with the two different tags, and there was a similar effect on mRNA levels of untagged LF receptor (co-transfected or endogenous), the down-regulation effect does not seem to be an artifact related to the protein tags employed. Downregulation of LF protein was specific to SF1b since co-transfection with untagged SFla or LF did not have the same effect. It seemed likely therefore that the result was not an artifact of overloading the protein translational machinery. However, since transfection with the same amount of cDNA does not result in the same amount of protein for each receptor type (Tan et al. 2005), we also employed a protein-expressing irrelevant plasmid to keep both total transfected DNA constant and to increase the load on protein synthesis in the absence of SF1b. Thus, we are confident that the effect of SF1b is not an artifact of over-taxation of the protein synthetic machinery under conditions of overexpression.

Having established that SF1b decreased the amount of receptor, we next began to address the mechanism. We considered the possibility that expression of SF1b increased degradation of the LF receptor. Our results from the hygromycin experiment suggest that SF1b has very little, if any, effect on the degradation of already formed protein, at least within the 24 -h period studied. It would be difficult to analyze the effect much beyond 
this since the general health of the cells would be compromised. These results are in general agreement with those from the Dufau lab (Qazi et al. 2006), which showed a similar rate of loss of radiolabeled LF in the presence and absence of SF1b. Given a decrease in protein and essentially no effect on degradation, the next logical experiment was to look at levels of mRNA.

Analysis by RT-PCR in the co-transfection system showed a 1:1 ratio of SF1b to LF cDNA to reduce the expression of LF mRNA by about $90 \%$ after $48 \mathrm{~h}$. This experiment also demonstrated that the effect was specific to SF1b since it did not occur with SFla. The result is not related to the expression vector since both SF receptor cDNAs were carried in the same parent vector.

An effect of SF1b cDNA on LF cDNA does not mean that SF1b cDNA would necessarily have this effect on the endogenous gene since there are so many more regulatory steps involved in processing the normal gene product. However, analysis of the effect of transfected SF1b cDNA on endogenous LF expression in the breast cancer cells demonstrated a similar decrease in LF expression, although the degree of effect was reduced due to differences in transfection efficiency between 293 cells and the breast cancer lines.

Because the effect is seen when both LF and SF1b are expressed through the use of plasmids, both of which employ the same strong CMV promoter, it is unlikely that any part of the effect on mRNA levels is transcriptional in nature. Therefore, to determine the mechanism whereby the decrease in steady state mRNA levels was achieved, we explored the possibility that expression of SF1b affected LF mRNA stability. The results demonstrated a major effect on mRNA stability since co-expression of SF1b accelerated LF mRNA degradation two- to three-fold. Because the untranslated regions would be different between the vector and endogenous mRNA, and yet the effect of SF1b was the same for transfected and endogenous, it seems most likely that this effect is either via the polyA tail (Meyer et al. 2004) or a microRNA mechanism targeting the translated region (Tay et al. 2008).

In order to be able to demonstrate the essential antagonism between the effects of LF and SF1b expression in cells which normally express PRL receptors, we transfected either additional LF alone or LF with SF1b and measured the result on viable cell number 5 days later. The experiment was conducted in this way in order to be able to titrate amounts of cDNA against one another and to correct for transfection efficiency. In other words, the response with transfected additional LF alone is representative of the proportion of cells transfected, and we can measure the ability of one-tenth or equal amounts of SF1b cDNA to counteract that response in an equivalent subpopulation. Thus, a $50 \%$ inhibition occurred at a cDNA ratio of LF to SF1b of 1:0 1 and complete inhibition occurred at 1:1 ratio. This correlates very nicely with the effect on protein levels measured with the luciferase-tagged LF, which is sufficiently quantitative for us to attempt such comparisons. These two experiments also show the dose-relatedness of the response since both doses were significantly different from control and different from each other.

The degree to which SF1b cDNA counteracts the effect of the LF cDNA in our studies is similar in magnitude to that reported for the rat SF PRL receptor (Berlanga et al. 1997) and to that reported by the Vonderhaar laboratory for SF1b (Trott et al. 2003). However, this differs from the efficacy reported for the SF1b construct produced by the Dufau laboratory where an eightfold amount of SF1b cDNA is required to quench the effect of LF cDNA (Qazi et al. 2006). The reason for this discrepancy is unclear at present.

The effect on mRNA and therefore levels of LF receptor that we describe here may not have been observed by the Dufau group because they only examined cell surface receptor (Qazi et al. 2006). In an overexpression system, only a proportion of receptors are on the surface and one would anticipate that quantities of cell surface receptor would be the last to show a reduction. However, in contrast, the Vonderhaar group did note a decrease in PRL binding sites in cells transfected such that they express both LF and SF1b versus those expressing LF alone (Trott et al. 2003).

In a very important study examining the relative expression of the LF and SF receptors in normal and tumorous breast tissue (including epithelium and stroma), the Dufau group quantified the ratio of LF to SF1b in normal breast tissue as $\sim 100: 1$ and in tumorous tissue as 1000:1 (Meng et al. 2004). The implication is that the reduced relative expression of the SF1b contributes in some way to tumor cell proliferation/ survival and is the reason we examined viable T47D cell number as a function of transfection with LF cDNA versus $\mathrm{LF}$ with SF1b cDNA. In the normal mammary epithelial cell line, MCF10A, these investigators also showed that the LF:SF1b ratio was $\sim 10: 1$ and that in a breast cancer cell line it was $\sim 100: 1$ (Meng et al. 2004). Our experiments with the 10:1 ratio of LF to SF1b are therefore reasonable for analysis of potentially physiologically/pathophysiologically relevant phenomena and are confirmed by exaggeration of the response by transfection with equal amounts of the two cDNAs. Our results on T47D cell number are therefore consistent with the hypothesis that the increased LF to SF1b ratio may contribute to tumor growth or tumor cell survival. Antagonism of signaling through the LF, either by LF-SF 1b heterodimerization or by down-regulation of LF expression, could counteract unmodified PRLstimulated promotion of the cell cycle (Brockman \& Schuler 2005) or anti-apoptotic signaling (LaVoie \& Witorsch 1995, Peierce \& Chen 2004). The source of this PRL could be either endocrine or autocrine 
(Ben-Jonathan et al. 2002), or both. In addition to antagonizing the growth-promoting effects of LF, we have previously shown that SF1b itself signals to increase expression of the vitamin $\mathrm{D}$ receptor and the cell cycle regulating protein, p21 (Wu et al. 2005).

The current results do not allow extrapolation to other species, in part because there are two short PRL receptors in the human, three in the mouse and only one in the rat (Ben-Jonathan et al. 2008); the role of individual short receptors may therefore be different.

In summary, we have identified an additional mechanism through which the SF1b receptor can act as a dominant negative. We have also demonstrated that tumor cell number is affected by physiologically relevant ratios of $\mathrm{SF} 1 \mathrm{~b}$ to $\mathrm{LF}$ receptors.

\section{Declaration of interest}

A M Walker is the inventor listed on US patent \# 6890738 which covers the use of S179D PRL.

\section{Funding}

This work was supported by California Breast Cancer Research Program grant 10PB-0127.

\section{Acknowledgements}

The authors thank Dr Daniel Jeske and the graduate students of the Statistics Consulting Collaboratory, particularly Yue Liu, for their assistance with data analysis.

\section{References}

Ben-Jonathan N, Liby K, McFarland M \& Zinger M 2002 PRL as an autocrine/paracrine growth factor in human cancer. Trends in Endocrinology and Metabolism 13 245-250.

Ben-Jonathan N, LaPensee CR \& LaPensee EW 2008 What can we learn from rodents about prolactin in humans? Endocrine Reviews 29 1-41.

Berlanga JJ, Garcia-Ruiz JP, Perrot-Applanat M, Kelly PA \& Edery M 1997 The short form of the prolactin (PRL) receptor silences PRL induction of the beta-casein gene promoter. Molecular Endocrinology 11 1449-1457.

Brockman J1 \& Schuler LA 2005 Prolactin signals via Stat5 and Oct-1 to the proximal cyclin D1 promoter. Molecular and Cellular Endocrinology 239 45-53.

Chen TJ, Kuo CB, Tsai KF, Liu JW, Chen DY \& Walker AM 1998 Development of recombinant human prolactin receptor antagonists by molecular mimicry of the phosphorylated hormone. Endocrinology 139 609-616.

Clevenger CV, Rycyzyn MA, Syed F \& Kline JB 2001 Prolactin receptor signal transduction. In Prolactin, pp 355-379. Ed ND Horseman. Boston: Kluwer Academic publishers.

Cosman D 1993 The hematopoietin receptor superfamily. Cytokine $\mathbf{5}$ 95-106.

Gadd SL \& Clevenger CV 2006 Ligand-independent dimerization of the human prolactin receptor isoforms: functional implications. Molecular Endocrinology 20 2734-2746.

Grotzinger J 2002 Molecular mechanisms of cytokine receptor activation. Biochimica et Biophysica Acta 1592 215-223.
Hu ZZ, Meng J \& Dufau ML 2001 Isolation and characterization of two novel forms of the human prolactin receptor generated by alternative splicing of newly identified exon 11. Journal of Biological Chemistry 276 41086-41094.

Huang Kuang Tzu, Chen Yen Hao \& Walker Ameae M 2004 Inaccuracies in MTS assays: major distorting effects of medium, serum albumin, and fatty acids. BioTechniques 37 406-412.

Kline JB, Roehrs H \& Clevenger CV 1999 Functional characterization of the intermediate isoform of the human prolactin receptor. Journal of Biological Chemistry 274 35461-35468.

LaVoie HA \& Witorsch RJ 1995 Investigation of intracellular signals mediating the anti-apoptotic action of prolactin in Nb2 lymphoma cells. Proceedings of the Society for Experimental Biology and Medicine 209 257-269.

Leav I, Merk FB, Lee KF, Loda M, Mandoki M, McNeal JE \& Ho SM 1999 Prolactin receptor expression in the developing human prostate and in hyperplastic, dysplastic, and neoplastic lesions. American Journal of Pathology 154 863-870.

Lee KF, Atiee SH \& Rosen JM 1989 Differential regulation of rat $\beta$-casein-chloramphenicol acetyltransferase fusion gene expression in transgenic mice. Molecular and Cellular Biology 9 560-565.

Li H, Ahonen TJ, Alanen K, Xie J, LeBaron MJ, Pretlow TG, Ealley EL, Zhang Y, Nurmi M, Singh B et al. 2004 Activation of signal transducer and activator of transcription 5 in human prostate cancer is associated with high histological grade. Cancer Research 64 $4774-4782$.

Meng J, Tsai-Morris CH \& Dufau ML 2004 Human prolactin receptor variants in breast cancer:low ratio of short forms to the long form human prolactin receptor associated with mammary carcinoma. Cancer Research 64 5677-5682.

Meyer S, Temme C \& Wahle E 2004 Messenger RNA turnover in eukaryotes: pathways and enzymes. Critical Reviews in Biochemistry and Molecular Biology 39 197-216.

Peirce SK \& Chen WY 2004 Human prolactin and its antagonist, hPRLG129R, regulate bax and bcl-2 gene expression in human breast cancer cells and transgenic mice. Oncogene 23 1248-1255.

Qazi AM, Tsai-Morris CH \& Dufau ML 2006 Ligand-independent homo- and heterodimerization of human prolactin receptor variants: inhibitory action of the short forms by heterodimerization. Molecular Endocrinology 20 1912-1923.

Tan D, Johnson DA, Wu W, Zeng L, Chen YH, Chen WY, Vonderhaar BK \& Walker AM 2005 Unmodified prolactin (PRL), and S179D PRL-initiated bioluminescence resonance energy transfer between homo- and hetero-pairs of long and short PRL receptors in living human cells. Molecular Endocrinology 19 1291-1303.

Tan D, Huang KT, Ueda E \& Walker AM 2008 S2 deletion variants of human prolactin receptors demonstrate that extracellular domain conformation can alter conformation of the intracellular signaling domain. Biochemistry 47 479-489.

Tay Y, Zhang J, Thomson AM, Lim B \& Rigoutsos I 2008 MicroRNAs to Nanog, Oct4 and Sox2 coding regions modulate embryonic stem cell differentiation. Nature 455 1124-1128.

Trott JF, Hovey RC, Koduri S \& Vonderhaar BK 2003 Alternative splicing to exon 11 of human prolactin receptor gene results in multiple isoforms including a secreted PRL-binding protein. Journal of Molecular Endocrinology 30 31-47.

Walker AM 2005 Prolactin receptor antagonists. Current Opinion in Investigational Drugs 6 378-385.

Wu W, Ginsburg E, Vonderhaar BK \& Walker AM 2005 S179D prolactin increases vitamin D receptor and p21 through upregulation of short $1 \mathrm{~b}$ prolactin receptor in human prostate cancer cells. Cancer Research 65 7509-7515.

Received 19 October 2009

Accepted 10 November 2009

Made available online as an Accepted Preprint 10 November 2009 